\title{
Construção de Manual de Orientações para a Educação Permanente em Centro Cirúrgico: relato de experiência
}

\author{
Construction of a Manual of Guidelines for Permanent Education in a Surgical Center: an \\ experience report
}

\section{Construcción de un Manual de Directrices para la Educación Permanente en un Centro Quirúrgico: un relato de experiencia}

Daniele Lima dos Anjos Reis ${ }^{1 *}$, Kátia Simone Kietzer ${ }^{1}$, Renata Campos de Sousa Borges ${ }^{1}$, Milena Coelho Fernandes Caldato ${ }^{1}$, Thiago Marcírio Gonçalves de Castro¹.

\begin{abstract}
RESUMO
Objetivo: Relatar a construção de uma tecnologia educativa, do tipo manual, que visa prestar orientações para o planejamento e implementação de ações de Educação Permanente no Centro Cirúrgico de um Hospital Público no interior do Estado do Pará, com ênfase na equipe de enfermagem. Relato de experiência: Intitulado "Manual de Orientações para a Educação Permanente em Centro Cirúrgico", esta tecnologia educativa fundamentou-se nos conceitos da Educação Permanente em Saúde (EPS) e das Metodologias Ativas de Ensino-Aprendizagem, buscando evidenciar a relevância da relação entre eles ao associá-los à assistência à saúde, especialmente no que se refere à assistência de enfermagem perioperatória em Centro Cirúrgico. Como resultados, evidencia-se a descrição detalhada da construção do manual, o qual foi dividido, estruturalmente, em 2 unidades: I - Sobre o que iremos falar? E II - Proposta para a execução do Manual de Orientações para a Educação Permanente em Centro Cirúrgico. Considerações finais: Este manual possui o potencial de instrumentalizar gestores responsáveis pela implementação dos processos de EPS no planejamento e na execução de ações educacionais aos profissionais que atuam em Centro Cirúrgico, com ênfase na equipe de enfermagem, estimulando o pensamento crítico-reflexivo.
\end{abstract}

Palavras-chave: Capacitação, Educação permanente, Enfermagem, Centro cirúrgico.

\begin{abstract}
Objective: To report the construction of an educational technology, of the manual type, which aims to provide guidelines for the planning and implementation of Permanent Education actions in the Surgical Center of a Public Hospital in the interior of the State of Pará, with emphasis on the nursing team. Experience report: Entitled "Manual of Guidelines for Permanent Education in a Surgical Center", this educational technology was based on the concepts of Permanent Education in Health (EPS) and Active Teaching-Learning Methodologies, seeking to highlight the relevance of the relationship between them by associating them with health care, especially with regard to perioperative nursing care in the Surgical Center. The results show the detailed description of the construction of the manual, which was structurally divided into 2 units: I - What are we going to talk about? E II - Proposal for the execution of the Manual of Guidelines for Permanent Education in a Surgical Center. Final considerations: This manual has the potential to equip managers responsible for implementing EPS processes in the planning and execution of educational actions for professionals working in the Surgical Center, with an emphasis on the nursing team, encouraging critical-reflective thinking.
\end{abstract}

Key words: Training, Permanent education, Nursing, Surgical center.

\section{RESUMEN}

Objetivo: Informar la construcción de una tecnología educativa, de tipo manual, que tiene como objetivo brindar lineamientos para la planificación e implementación de acciones de Educación Permanente en el Centro Quirúrgico del Hospital Público del interior del Estado de Pará, con énfasis en la equipo de enfermería. Informe de experiencia: Titulado "Manual de Lineamientos para la Educación Permanente en un Centro Quirúrgico", esta tecnología educativa se basó en los conceptos de Educación Permanente en Salud (EPS) y

1 Universidade do Estado do Pará (UEPA), Tucuruí - PA. *E-mail: anjo.daniele@hotmail.com SUBMETIDO EM: 2/2021 
Metodologías Activas de Enseñanza-Aprendizaje, buscando resaltar la relevancia de la relación entre ellos asociándolos a los cuidados de salud, especialmente en lo que respecta a los cuidados de enfermería perioperatorios en el Centro Quirúrgico. Los resultados muestran la descripción detallada de la construcción del manual, que se dividió estructuralmente en 2 unidades: I - ¿De qué vamos a hablar? E II - Propuesta para la ejecución del Manual de Lineamientos para la Educación Permanente en un Centro Quirúrgico. Consideraciones finales: Este manual tiene el potencial de dotar a los gerentes responsables de implementar procesos de EPS en la planificación y ejecución de acciones educativas para los profesionales que laboran en el Centro Quirúrgico, con énfasis en el equipo de enfermería, fomentando el pensamiento crítico-reflexivo.

Palabras clave: Formación, Educación permanente, Enfermería, Centro quirúrgico.

\section{INTRODUÇÃO}

A Educação Permanente é considerada uma medida capaz de transformar as práticas educativas nos campos da formação, atenção, gestão, formação de políticas, participação popular e controle social no setor saúde (BRASIL, 2018a).

O termo Educação Permanente em Saúde (EPS) refere-se a um processo de aprendizagem que possibilita a construção de conhecimentos a partir de situações vivenciadas no trabalho (BRASIL, 2018b). Nela, as temáticas estudadas são escolhidas com base nos problemas do cotidiano, de forma que a necessidade de conhecimento que emerge da prática leva à mudanças na formação e no desenvolvimento do profissional, colocando-o no centro do processo ensino-aprendizagem (GROSSI MG e KOBAYASHI RM, 2013). Assim, a aprendizagem só se torna significativa quando corresponde a um desejo do indivíduo (PARÁ, 2017).

No Brasil, os processos de Educação Permanente foram instituídos pela Portaria GM/MS no 198/2004, sendo alterada pela Portaria GM/MS no 1996/2007, que apresentou novas estratégias e diretrizes para a implementação da Política Nacional de Educação Permanente em Saúde (PNEPS) (BRASIL, 2009).

Cotrim-Guimarães IMA (2009) refere que o desenvolvimento dos processos de EPS exige a adoção de metodologias ativas de aprendizagem, que considerem a articulação entre teoria e prática, a problematização da realidade, a participação ativa dos profissionais e atores sociais envolvidos, configurando-se num processo de aprendizagem mútua e significativa. Sobre isso, afirma-se que metodologias ativas são estratégias de ensino-aprendizagem compostas por métodos e técnicas que utilizam experiências reais ou simuladas com o intuito de instigar a busca de conhecimento (SALVADOR CAB, et al., 2019).

O uso das metodologias ativas com o aporte de tecnologias educativas propicia aos profissionais de saúde o desenvolvimento do papel de orientador e facilitador de maneira mais criativa e ampla, fomentando processos educativos no contexto da EPS realmente significativos (DIAS SR e VOLPATO AN, 2017; NIETSCHE EA, et al., 2012). As tecnologias educativas são aquelas utilizadas para mediação de processos de ensinar e aprender nos processos de ensino-aprendizagem (TEIXEIRA E e MOTA VMSS, 2011).

A Educação Permanente em Enfermagem no Centro Cirúrgico tem o objetivo de propiciar aos profissionais conhecimentos técnicos específicos e o desenvolvimento de habilidades para uma assistência de maior qualidade e eficácia, considerando as especificidades do setor, o que gera desenvolvimento pessoal e profissional (RIBEIRO MB, et al., 2011).

A responsabilidade das instituições hospitalares em colaborar com a execução da PNEPS está ancorada na Portaria de Consolidação no 2, de 28 de setembro de 2017, que trata da Consolidação das normas sobre as políticas nacionais de saúde do SUS, e traz em seus artigos $8^{\circ}$ e 9ํㅜㄹ incisos XII e IV, respectivamente, as competências de assegurar o desenvolvimento de educação permanente para seus trabalhadores e de ser campo de educação permanente para profissionais das Redes de Atenção à Saúde, conforme pactuado com o gestor público de saúde local (BRASIL, 2017).

Desta forma, considerando que as práticas educacionais ainda são insipientes no Centro Cirúrgico de um Hospital Público do interior do estado do Pará e compreendendo a necessidade de qualificação dos profissionais de enfermagem que lá atuam, surgiu a necessidade de propor novas ações de EPS, substituindo 
as práticas esporádicas por práticas contínuas e sistematizadas e os métodos educacionais em que o facilitar é um mero transmissor de conhecimentos por métodos que propiciem a aprendizagem significativa, fortalecendo a problematização e o pensamento crítico-reflexivo dos envolvidos no processo (FUNESA, 2011). Considerando o exposto, este estudo propõe relatar a construção de uma tecnologia educativa, do tipo manual, que visa prestar orientações para o planejamento e implementação de ações de Educação Permanente no Centro Cirúrgico de um Hospital Público, com ênfase na equipe de enfermagem.

\section{RELATO DE EXPERIÊNCIA}

Trata-se de um estudo descritivo, do tipo relato de experiência, como parte de uma dissertação do Mestrado Profissional Ensino em Saúde na Amazônia, por uma universidade pública no estado do Pará. Trabalhos do tipo relato de experiência são considerados uma ferramenta da pesquisa descritiva pautada em uma reflexão de ações que se referem a situações vivenciadas no âmbito profissional de interesse da comunidade científica (PEREIRA MO, et al., 2020).

Para a construção do presente trabalho, inicialmente foi realizada uma pesquisa de campo, de abordagem quantitativa e caráter descritivo, sendo aprovada pelo Comitê de Ética em Pesquisa da Universidade do Estado do Pará (UEPA), sob o número do Parecer: 3.137.684. Os resultados desta embasaram a construção do Manual de Orientações. Tal construção ocorreu entre os meses de dezembro de 2020 e janeiro de 2021 e se deu através de 04 etapas:

1aㅡ etapa: Criação do Grupo de Trabalho, composto por profissionais e acadêmicos da área da saúde envolvidos com os processos temáticos à Centro Cirúrgico, sendo 12 enfermeiros, 1 fisioterapeuta, 02 médicos e 09 acadêmicos do curso de Graduação em Enfermagem de uma universidade pública do interior do estado do Pará.

$2^{\text {a }}$ etapa: Reunião com o Grupo de Trabalho, para discussão da temática; delimitação das unidades e seções e definições de layout, responsabilidades e prazos;

3aㅡ etapa: Construção Teórica do Manual de Orientações, por meio de pesquisas bibliográficas de artigos disponíveis na Biblioteca Virtual em Saúde (BVS), nas seguintes bases de dados: Scientific Electronic Library Online (Scielo) e Literatura Latino-Americana e do Caribe em Ciências da Saúde (Lilacs); bem como por meio de instrumentos como livros didáticos e manuais/protocolos sobre as diversas temáticas abordadas.

4aㅡ etapa: Execução da Diagramação, na qual priorizou-se a atratividade e o manuseio facilitado pelo leitor. Nesta etapa, os conteúdos foram organizados no editor de texto do Microsoft Word e, posteriormente, encaminhados à editora responsável pela editoração e publicação da tecnologia.

A tecnologia educativa elaborada foi intitulada "Manual de Orientações para a Educação Permanente em Centro Cirúrgico" e debruçou-se sobre os conceitos da Educação Permanente em Saúde (EPS) e das Metodologias Ativas de Ensino-Aprendizagem, buscando evidenciar a inegável relevância da relação entre eles ao associá-los à assistência à saúde, especialmente no que se refere à assistência de enfermagem perioperatória em Centro Cirúrgico.

Estruturalmente, a parte inicial do Manual foi organizada com os seguintes tópicos: capa, contracapa, conselho editorial, ficha catalográfica, dedicatória, prefácio, organizadoras, colaboradores, apresentação e sumário. Além disso, foi dividido em 2 unidades consideradas complementares e interdependentes, baseadas na comunicação dialógica e considerando as experiências do público-alvo das ações propostas. Ao final, foram apresentados os anexos e o índice remissivo. A seguir são detalhadas as duas unidades que compõem o Manual.

\section{Unidade I: sobre o que iremos falar?}

Esta unidade constituiu-se de 4 capítulos, que remetem a reflexão a respeito das bases conceituais e metodológicas do âmago desta obra: a Educação Permanente, evidenciando o seu diagnóstico no Centro Cirúrgico do hospital de realização do estudo que subsidiou a elaboração deste manual, bem como as necessidades de aprendizagem da equipe de enfermagem que atua neste setor (Quadro 1). 
Quadro 1 - Capítulos da Unidade I do Manual.

\begin{tabular}{|c|c|c|}
\hline Capítulo & Temática & Abordagem \\
\hline I & O objeto de estudo deste Manual & $\begin{array}{l}\text { Buscou evidenciar o objeto de estudo do Manual: a } \\
\text { Educação Permanente }\end{array}$ \\
\hline II & $\begin{array}{l}\text { Diagnóstico Situacional da } \\
\text { Educação Permanente no Centro } \\
\text { Cirúrgico do HRT }\end{array}$ & $\begin{array}{l}\text { Buscou apontar a realidade atual do Centro Cirúrgico do } \\
\text { HRT em relação aos processos de Educação } \\
\text { Permanente, traçando o perfil dos profissionais de } \\
\text { enfermagem do setor (categoria profissional, sexo, faixa } \\
\text { etária, tempo de atuação no setor); as práticas de } \\
\text { Educação Permanente realizadas e as possibilidades } \\
\text { para a Educacão Permanente }\end{array}$ \\
\hline III & $\begin{array}{ll}\text { Educação } & \text { Permanente } \quad \text { em } \\
\text { Saúde: bases legais e conceituais }\end{array}$ & $\begin{array}{l}\text { Buscou apresentar um cunho teórico-metodológico a } \\
\text { respeito dos processos de EPS }\end{array}$ \\
\hline IV & $\begin{array}{l}\text { Levantamento das necessidades } \\
\text { de aprendizagem da equipe de } \\
\text { enfermagem }\end{array}$ & $\begin{array}{l}\text { Buscou desvelar as necessidades de } \\
\text { treinamentos/capacitações da equipe de enfermagem } \\
\text { do setor, a fim de efetivar os preceitos da EPS, que } \\
\text { valoriza a aprendizagem a partir de situações } \\
\text { problemáticas vivenciadas no cotidiano laboral }\end{array}$ \\
\hline
\end{tabular}

Fonte: Reis DLA, et al., 2021.

Destaca-se, nesse contexto, o levantamento das necessidades de aprendizagem da equipe de enfermagem do setor, evidenciada pela aplicação de uma ferramenta de gestão denominada "Matriz GUT", utilizada para priorizar ações dentro de um conjunto de alternativas, tendo como objetivo ordenar a importância delas pela sua GRAVIDADE (impacto do problema sobre as coisas, pessoas, resultados ou processos), URGÊNCIA (relação com o tempo disponível ou necessário para resolução do problema) e TENDÊNCIA (potencial de crescimento ou desaparecimento do problema), de forma racional (COSTA ARS, et al., 2017).

Foram apresentados problemas identificados no cotidiano laboral em Centro Cirúrgico e os participantes da pesquisa realizada inicialmente foram orientados para preenchimento dos campos $G$ (gravidade), $U$ (urgência) e $T$ (tendência), pontuando através da utilização de uma escala que vai de 1 a 5 , sempre na tentativa de responder aos seguintes questionamentos: Qual a gravidade do problema? Qual a urgência de se eliminar o problema? Qual a tendência e potencial de crescimento do problema?

O resultado da multiplicação entre as 3 notas obtidas para cada problema ( $G \times U \times T)$ correspondeu ao total de pontuação, o que possibilitou a priorização dos problemas identificados. Para se obter uma tabela totalizadora, foram somados os escores apontados por cada participante, de cada problema em relação a cada categoria, sendo extraída a média, conforme demonstrado no Quadro 2.

Quadro 2 - Problemas identificados e ordem de priorização de resolução.

\begin{tabular}{|c|c|c|c|c|c|}
\hline Problema identificado & G & $\mathbf{U}$ & $\mathbf{T}$ & Total & Priorização \\
\hline Não utilização do Checklist de Cirurgia Segura & 5 & 5 & 5 & 125 & $1^{0} \stackrel{0}{ }$ \\
\hline Infecção em centro cirúrgico & 5 & 5 & 5 & 125 & $1^{0}$ \\
\hline $\begin{array}{l}\text { Conhecimento incipiente sobre condutas durante uma parada } \\
\text { cardiorrespiratória }\end{array}$ & 5 & 5 & 4 & 100 & $2^{0}$ \\
\hline $\begin{array}{l}\text { Baixa padronização no uso dos instrumentais e montagem da mesa de } \\
\text { instrumentação cirúrgica }\end{array}$ & 5 & 5 & 4 & 100 & $2^{o}$ \\
\hline Conhecimento incipiente sobre condutas emergenciais ao Recém-Nascido & 4 & 4 & 4 & 64 & 3은 \\
\hline Desajustes na montagem e preparo da sala operatória & 3 & 4 & 4 & 48 & $4^{\circ}$ \\
\hline Mecanização da assistência & 4 & 4 & 3 & 48 & $4^{0}$ \\
\hline Anotações de enfermagem ineficientes & 5 & 3 & 3 & 45 & $5^{0}$ \\
\hline $\begin{array}{l}\text { Necessidade de utilização de posições cirúrgicas mais adequadas e } \\
\text { confortáveis ao paciente }\end{array}$ & 4 & 3 & 3 & 36 & $6^{\circ}$ \\
\hline Conhecimento incipiente sobre classificação cirúrgica & 4 & 3 & 3 & 36 & 60 \\
\hline Conhecimento incipiente sobre anestesiologia básica & 3 & 3 & 3 & 27 & $7^{0}$ \\
\hline $\begin{array}{l}\text { Conhecimento incipiente sobre condutas na Sala de Recuperação Pós- } \\
\text { Anestésica (SRPA) }\end{array}$ & 3 & 2 & 3 & 18 & $8^{\circ}$ \\
\hline
\end{tabular}

Fonte: Reis DLA, et al., 2021. 


\section{Unidade II: proposta para a execução do manual de orientações para a educação permanente em centro cirúrgico}

Partindo da análise dos problemas elencados anteriormente, foi possível elaborar um plano de capacitações com temas relativos às problemáticas apresentadas, obedecendo a prioridade de resolução.

Assim, esta unidade constituiu-se de 13 capítulos, seguindo a numeração sequencial da unidade anterior, nos quais foram apresentadas estratégias inovadoras para a abordagem de diversos temas relativos à Educação Permanente em Centro Cirúrgico, considerando métodos ativos de ensino-aprendizagem (Quadro 3).

Quadro 3 - Capítulos da Unidade II do Manual.

\begin{tabular}{|c|l|}
\hline Capítulo & \multicolumn{1}{|c|}{ Temática } \\
\hline $\mathrm{V}$ & Apresentação dos temas para capacitações \\
\hline $\mathrm{VI}$ & Segurança do paciente cirúrgico \\
\hline $\mathrm{VII}$ & Controle de infecção e Biossegurança em Centro Cirúrgico \\
\hline $\mathrm{VIII}$ & Assistência de enfermagem durante parada cardiorrespiratória \\
\hline $\mathrm{IX}$ & Instrumentação cirúrgica \\
\hline $\mathrm{X}$ & Assistência de enfermagem nos cuidados emergenciais ao Recém-nascido \\
\hline $\mathrm{XI}$ & Montagem da sala operatória \\
\hline $\mathrm{XII}$ & Humanização da assistência em Centro Cirúrgico \\
\hline $\mathrm{XIII}$ & Anotações de enfermagem no período perioperatório \\
\hline $\mathrm{XIV}$ & Posicionamento cirúrgico e prevenção de lesões por pressão \\
\hline $\mathrm{XV}$ & Classificação Cirúrgica \\
\hline $\mathrm{XVI}$ & Anestesiologia Básica \\
\hline$X V I I$ & Assistência de enfermagem na Sala de Recuperação Pós-Anestésica \\
\hline
\end{tabular}

Fonte: Reis DLA, et al., 2021.

O quadro acima evidencia a listagem de temas das capacitações explicitadas pelo Manual, considerando, efetivamente, a opinião do público-alvo. Assim, para melhor abordagem dos temas, definiu-se um padrão metodológico organizacional para cada capítulo da Unidade II, iniciando pelo capítulo VI (Quadro 4).

Quadro 4 - Padrão metodológico dos capítulos pertencentes à Unidade II do Manual.

\begin{tabular}{|l|l|}
\hline Padrão metodológico dos capítulos & \multicolumn{1}{|c|}{ Abordagem } \\
\hline Introdução & $\begin{array}{l}\text { Buscou apresentar uma breve explanação sobre o objeto de } \\
\text { estudo do capítulo }\end{array}$ \\
\hline Objetivo & Buscou evidenciar a finalidade do capítulo \\
\hline Temática da capacitação & $\begin{array}{l}\text { Buscou explanar conceitualmente o assunto foco da } \\
\text { abordagem pela capacitação sugerida }\end{array}$ \\
\hline Público-alvo & $\begin{array}{l}\text { Buscou apontar a qual (ou quais) grupo(s) a capacitação foi } \\
\text { sugerida }\end{array}$ \\
\hline Local de realização & $\begin{array}{l}\text { Buscou sugerir o local mais apropriado para a execução da } \\
\text { capacitação }\end{array}$ \\
\hline $\begin{array}{l}\text { Proposta de execução da ação: } \\
\text { - Estratégias educacionais sugeridas para a a a ardagem } \\
\text { - Descrição da execução da ação } \\
\text { - Materiais necessários } \\
\text { - Carga horária } \\
\text { - Periodicidade da execução da ação } \\
\text { - Estratégias de avaliação da ação }\end{array}$ & $\begin{array}{l}\text { Buscou detalhar, metodologicamente, as estratégias de ação } \\
\text { educativa sugeridas, sempre na tentativa de associar os } \\
\text { processos de Educação Permanente com o uso de métodos } \\
\text { ativos de aprendizagem }\end{array}$ \\
\hline Resumo do capítulo & $\begin{array}{l}\text { Buscou mostrar, de forma didática, objetiva e ilustrativa, } \\
\text { através de um organograma, um resumo dos conteúdos } \\
\text { abordados pelo capítulo }\end{array}$ \\
\hline Referências & $\begin{array}{l}\text { Buscou listar a literatura consultada para a construção do } \\
\text { capítulo }\end{array}$ \\
\hline
\end{tabular}

Fonte: Reis DLA, et al., 2021. 


\section{DISCUSSÃO}

A Unidade de Centro Cirúrgico é um conjunto de elementos destinados à atividade cirúrgica e à recuperação anestésica e pós-operatória imediata (CARVALHO R e BIANCHI ERF, 2016). Este setor é considerado um ambiente único e diferenciado dentro do ambiente hospitalar, devendo dispor de uma série de requisitos que o torna apto à realização do procedimento cirúrgico. Nele realizam-se técnicas estéreis que garantem a segurança do paciente atendido quanto ao controle de infecção (SOBECC, 2017).

Vale registrar que a contínua transformação oriunda dos avanços tecnológicos tem revolucionado as questões relacionadas ao ambiente cirúrgico no que se refere a materiais, equipamentos, técnicas e procedimentos, exigindo que a equipe de enfermagem que atua neste âmbito esteja sempre capacitada, atualizada e apta a prestar assistência de qualidade e com responsabilidade aos pacientes cirúrgicos (RIBEIRO MB, et al., 2011).

Verifica-se, assim, que para que haja transformação efetiva da qualidade dos serviços de saúde pública neste país é imprescindível a qualificação contínua dos profissionais que atuam no Sistema Único de Saúde (SUS), ganhando forças os debates a respeito dos processos de EPS, sendo este alvo de muitas discussões em âmbito nacional, que debatem a necessidade de atualização da PNEPS (GONÇALVES VMA, 2017).

Destarte, o processo de ensino-aprendizagem executado nas ações de Educação Permanente e mediado pelo uso de metodologias ativas favorece o desenvolvimento de competências concomitantemente à construção do conhecimento significativo (DIAS SR e VOLPATO AN, 2017). Assim, a integração entre teoria e prática fomentada pelos métodos ativos proporciona novas possibilidades de formação, que se faz mais sólida e coerente e torna efetiva a aprendizagem significativa, onde o educando é estimulado a construir o conhecimento ao invés de recebê-lo de forma passiva (PAIVA MRF, et al., 2016).

O uso de tecnologias na saúde tem sido cada vez mais referido quando se trata da implementação de ações relacionadas à Educação Permanente. Amplamente utilizadas pela enfermagem, as tecnologias educacionais referem-se à utilização dos recursos tecnológicos como ferramentas para aprimorar o ensino, consideradas instrumentos facilitadores para construção e reconstrução do conhecimento (NIETSCHE EA et al., 2012; PAES ED e COSTA CS, 2017).

Dentre os variados exemplos de tecnologias educacionais disponíveis, evidenciam-se os manuais educacionais, que surgem como ferramentas de auxílio às equipes de saúde, em especial o enfermeiro, por serem amplamente utilizados nos processos de EPS para apoiar a construção do conhecimento (NASCIMENTO CCL, et al., 2020).

Diante do exposto, ressalta-se a importância do levantamento das necessidades de capacitação de uma equipe para que seja possível a implementação dos processos de EPS de maneira eficaz. Tais demandas não devem limitar-se a uma lista de necessidades de aprendizagem individuais, mas sim partirem de problemas reais, vivenciados no dia a dia do trabalho (SILVA MF, et al., 2009).

Assim, os problemas elencados no Quadro 2 foram desvelados por meio de observações diretas e por relatos da equipe de enfermagem do setor, por meio da técnica de brainstorming não estruturado, na oportunidade de reuniões setoriais. Esta técnica é considerada uma estratégia assertiva, pois configura-se como uma boa estratégia para conhecer as percepções das pessoas que participam do momento, pois nessa metodologia as ideias são aleatoriamente expostas, à medida que o assunto evoluiu e se tem o momento mais oportuno para expor opiniões, além de deixar o ambiente mais relaxado e de fato considerar 0 posicionamento da equipe (FILHO MT e ALVES T, 2013).

Observou-se que o problema "Não utilização do Checklist de Cirurgia Segura", juntamente com "Infecção em Centro Cirúrgico" dividiram o 1 lugar na prioridade apontada pelos profissionais do setor, o que direcionou à proposta dos temas de capacitações "Segurança do paciente cirúrgico" e "Controle de infecção e Biossegurança em Centro Cirúrgico", apresentados no Quadro 3. Pode-se estabelecer uma relação entre os dois problemas, pois, a ausência de utilização de Checklist de Cirurgia Segura pode colaborar para o aumento de infecções de sítios cirúrgicos, considerando que o Checklist visa à redução de erros derivados da realização de procedimentos cirúrgicos. 
O Checklist de Cirurgia Segura foi proposto para ser aplicado em qualquer instituição hospitalar, independente da complexidade, pública ou privada, propiciando às equipes cirúrgicas seguir de forma sistemática a verificação de pontos críticos de segurança, visando uma avaliação integral do paciente e podendo ser adaptada para a realidade das instituições de saúde que a utilizam (SANTOS EA, et al., 2020).

Em relação ao controle das infecções, suscita-se a integração das medidas de biossegurança, que abrangem um conjunto de ações direcionadas aos profissionais, usuários e o ambiente da exposição aos riscos biológicos, bem como a escolha adequada de práticas para prevenir infecções (DEPARTAMENTO DO TRABALHO DOS EUA, 2012).

Considerando a relevância da construção e utilização de manuais educacionais na educação profissional, destaca-se a necessidade de organização dos conteúdos selecionados para compor este tipo de tecnologia educativa. Nesse sentido, afirma-se que os dados apresentados pelo Quadro 4 demonstra o interesse dos pesquisadores em contribuir para o melhor entendimento das temáticas sugeridas no plano de capacitações, facilitando, assim, a implementação efetiva de todas as estratégias ativas propostas.

Vale ressaltar que a avaliação dos processos educacionais deve ser considerada como estratégia relevante no decorrer das práticas de EPS, pois a proposta é que os profissionais de saúde, a partir do seu conhecimento prévio, dialoguem entre si sobre formas de enfrentamento das questões criando um ambiente de ensino-aprendizagem que faça sentido no cotidiano de suas práticas e não seja mera transmissão de conhecimentos, ou seja, onde haja aprendizagem significativa (ALAGOAS, 2018).

Os propósitos do PNEPS são fortalecidos à medida que ações educacionais estratégicas são implementadas na educação profissional, especialmente quando alinhadas aos alicerces dos métodos ativos de ensino-aprendizagem. Dessa forma, a utilização de tecnologias educativas, como o manual descrito neste trabalho, tem o potencial de instrumentalizar gestores responsáveis pelo planejamento e execução dos processos de EPS aos profissionais que atuam em Centro Cirúrgico, com ênfase nas equipes de enfermagem, estimulando o pensamento crítico-reflexivo.

\section{REFERÊNCIAS}

1. ALAGOAS. Secretaria de Estado da Saúde de Alagoas (SESAU/AL). Guia de Educação Permanente em Saúde (EPS). Gerência Executiva de Valorização de Pessoas (GEREVP), Gestão de Desenvolvimento e Educação em Saúde (GDES), Comissão de Integração Ensino Serviço Estadual. Maceió, Alagoas, 2018. Disponível em: http://www.educasesauead.saude.al.gov.br/pluginfile.php/1556/mod_resource/content/2/CARTILHA_EPS_ONLINE. pdf. Acessado em: 20 de janeiro de 2021.

2. BRASIL. Ministério da Saúde. Secretaria de Gestão do Trabalho e da Educação na Saúde. Departamento de Gestão da Educação na Saúde. Política Nacional de Educação Permanente em Saúde: o que se tem produzido para o seu fortalecimento? Brasília: Ministério da Saúde, 2018a. Disponível em: https://bvsms.saude.gov.br/bvs/publicacoes/politica_nacional_educacao_permanente_saude_fortalecimento.pdf. Acessado em: 20 de janeiro de 2021.

3. BRASIL. Ministério da Saúde. Secretaria de Gestão do Trabalho e da Educação na Saúde. Manual Técnico 2018Programa para o Fortalecimento das Práticas de Educação Permanente em Saúde no SUS - PRO EPS-SUS. Brasília: Ministério da Saúde, 2018b. Disponível em: https://portalarquivos2.saude.gov.br/images/pdf/2018/marco/28/ManualTecnico-PRO-EPS-SUS-MINUTA17-10.pdf. Acessado em: 21 de janeiro de 2021.

4. BRASIL. Ministério da Saúde. Gabinete do Ministro. Portaria de Consolidação oㅡ 2, de 28 de setembro de 2017. Consolidação das normas sobre as políticas nacionais de saúde do Sistema Único de Saúde. Brasília: Ministério da Saúde, 2017. Disponível em: http://bvsms.saude.gov.br/bvs/saudelegis/gm/2017/prc0002_03_10_2017.html. Acessado em: 21 de janeiro de 2021.

5. BRASIL. Ministério da Saúde. Secretaria de Gestão do Trabalho e da Educação na Saúde. Departamento de Gestão da Educação em Saúde. Política Nacional de Educação Permanente em Saúde. Brasília: Ministério da Saúde, 2009. Disponível em: https://bvsms.saude.gov.br/bvs/publicacoes/politica_nacional_educacao_permanente_saude.pdf. Acessado em: 21 de janeiro de 2021.

6. CARVALHO R, BIANCHI ERF. Enfermagem em Centro Cirúrgico e Recuperação. São Paulo: Manole, 2016.

7. COSTA ARS, et al. Aplicação da Matriz GUT na gestão integrada de resíduos sólidos da cidade do Recife - PE. Revista AIDIS, 2017;10(2): 201-213.

8. COTRIM-GUIMARÃES IMA. Programa de educação permanente e continuada da equipe de enfermagem da clínica médica do Hospital Universitário Clemente de Faria: análise e proposições. Dissertação (Mestrado em Saúde Pública), Escola Nacional de Saúde Pública Sergio Arouca, Rio de Janeiro, 2009; 149p. 
9. DEPARTAMENTO DO TRABALHO DOS EUA. Patógenos transmitidos pelo sangue. In: Normas de segurança e saúde ocupacional. 2012 OSHA.gov.

10. DIAS SR, VOLPATO AN. Práticas inovadoras em metodologias ativas. Florianópolis: Contexto Digital, 2017.

11. FILHO MT, ALVES T. Ferramentas para gestão de resultados. Brasília: Senac-DF, 2013.

12. FUNESA. Fundação Estadual de Saúde. Educação Permanente em Saúde no Estado de Sergipe: Saberes e Tecnologias para Implantação de uma Política. Livro do Aprendiz 2. Aracaju: FUNESA, 2011. Disponível em: https://www.funesa.se.gov.br/wp-content/uploads/2019/01/Educa\%C3\%A7\%C3\%A3o-Permanente-Volume2_aprendiz.pdf. Acessado em: 22 de janeiro de 2021.

13. GONÇALVES VMA. Educação permanente e parada cardiorrespiratória: um relato de experiência no âmbito da enfermagem. Gerais: Revista de Saúde Pública do SUS/MG, 2017; 2(2): 47-58.

14. GROSSI MG, KOBAYASHIA RM. A construção de um ambiente virtual de aprendizagem para educação a distância: uma estratégia educativa em serviço. Rev Esc Enferm USP, 2013;47(3): 756-760.

15. NASCIMENTO CCL, et al. Educação permanente em sala de imunização: elaboração de manual de normas e rotinas. Research, Society and Development, 2020; 9(8): e176985601.

16. NIETSCHE EA, et al. Tecnologias inovadoras do cuidado em enfermagem. Rev Enferm UFSM, 2012; 2(1): $182-189$.

17. PAES ED, COSTA CS. Guia do produto educacional Coisa de professor: compartilhamento de ideias e saberes em tecnologia educacional. Dissertação (mestrado). Colégio Pedro II, Rio de Janeiro, 2017.

18. PAIVA MRF, et al. Metodologias ativas de ensino-aprendizagem: revisão integrativa. SANARE, 2016; 15(2): 145 -153.

19. PARÁ. Secretaria de Estado de Saúde Pública. Diretoria de Gestão do Trabalho e Educação na Saúde. Coordenação de Educação na Saúde. Gerência de Educação Permanente. Política Nacional de Educação Permanente em Saúde: manual de orientações para projetos de formação em saúde. Belém: Sespa, 2017.

20. PEREIRA MO, et al. Superando os desafios para oferecer formação de qualidade em enfermagem psiquiátrica. Revista Brasileira de Enfermagem, 2020; 73(1):e20180208.

21. RIBEIRO MB, et al. Estratégias de capacitação da equipe de enfermagem de um centro cirúrgico oncológico. Rev SOBECC,2011; 16(03): 21-29.

22. SALVADOR CAB, et al. Simulação realística, estratégia metodológica para a formação de profissionais na área da saúde: uma revisão integrativa. Rev. Bra. Edu. Saúde, 2019; 9(4): 58-64.

23. SANTOS EA, et al. Surgical safety checklist: knowledge and challenges for the surgical center team. Revista Enfermería Actual, 2020; Edición Semestral № 38: 1-14

24. SILVA MF, et al. Educação continuada: um levantamento de necessidades da equipe de enfermagem. Arq Bras Ciên Saúde, 2009; 34(1): 15-21.

25. SOBECC. Sociedade Brasileira de Enfermeiros de Centro Cirúrgico, Recuperação Anestésica e Centro de Material e Esterilização. Diretrizes de práticas em enfermagem cirúrgica e processamento de produtos para a saúde. 7 ed. São Paulo: SOBECC, 2017.

26. TEIXEIRA E, MOTA VMSS. Tecnologias educacionais em foco. Série Educação em Saúde. São Caetano do Sul: Difusão Editora, 2011. 